\title{
Psycho-Education and Group Cognitive-Behavioural Therapy for Clinical Perfectionism: A Case-Series Evaluation
}

\author{
Anna L. Steele and Sue Waite \\ Adelaide Health Service, Australia \\ Sarah J. Egan, Janelle Finnigan and Alicia Handley \\ Curtin University, Australia \\ Tracey D. Wade \\ Flinders University, Australia
}

\begin{abstract}
Background: Research indicates that psycho-education and cognitive behavioural interventions can reduce perfectionism but to date no group treatments have been examined. Aims: The current study utilized a case series design to compare psycho-education materials and subsequent eight-week group cognitive behaviour therapy (CBT) to a baseline waitlist in an outpatient community psychiatry sample $(n=21)$. Method: Participants were assessed on five occasions: baseline, 4 weeks later (waitlist), 4 weeks after receiving psycho-education material, post-treatment ( 8 weeks after receiving the group intervention), and 3-month followup. Results: There was a main effect of time for perfectionism and negative affect from baseline to post-group (effect sizes ranging from 1.46 to 1.91) that were maintained at 3-month follow-up. Conclusions: These results suggested that group CBT for clinical perfectionism may be beneficial, but that psycho-education alone is not effective for reducing perfectionism or negative affect.
\end{abstract}

Keywords: Perfectionism, cognitive behaviour therapy.

\section{Introduction}

Perfectionism has been implicated as important in the aetiology and maintenance of depression, anxiety and eating disorders (Egan, Wade and Shafran, 2011). Higher scores on perfectionism compared to non-clinical controls have been found in individuals with obsessive-compulsive disorder (Frost and Steketee, 1997), social phobia (Juster et al., 1996) and panic disorder (Antony, Purdon, Huta and Swinson, 1998). Perfectionism has been found

Reprint requests to Sarah Egan, School of Psychology and Speech Pathology and Curtin Health Innovation Research Institute, Curtin University, GPO Box U1987, Perth, Western Australia, Australia 6847. E-mail: s.egan@ curtin.edu.au 
to be elevated in samples with clinical depression (Sassaroli et al., 2008) and predictive of suicidal ideation 6-months later in an inpatient setting (Beevers and Miller, 2004). Prospective studies have indicated perfectionism may predispose individuals to eating pathology (e.g. Tyrka, Waldron, Graber and Brooks-Gunn, 2002; Vohs, Bardone, Joiner, Abramson and Heatherton, 1999). Perfectionism also negatively affects treatment outcome in a number of ways: it interferes with an individual's ability to participate in tasks of exposure, response prevention and cognitive restructuring for obsessive-compulsive disorder (Frost, Novara and Rhéaume, 2002); it decreases response for social anxiety (Lundh and Ost, 2001); it is associated with less reduction in depressive symptoms at the end of treatment for depression and at follow-up (Blatt and Zuroff, 2005; Hawley, Ho, Zuroff and Blatt, 2006). Hence perfectionism has been represented as a transdiagnostic mechanism, a construct of interest across a range of psychopathologies (Egan et al., 2011).

The perfectionism construct has been defined in a number of ways, with earlier focus being on perfectionism as a multidimensional construct, predominantly measured by two Multidimensional Perfectionism Scales (MPS); the 35-item FMPS (Frost, Marten, Lahart, and Rosenblate, 1990) and the 45-item HMPS (Hewitt and Flett, 1991). The FMPS contains five subscales of pertinence to perfectionism: Personal Standards; Concern over Mistakes; Parental Expectations; Parental Criticism; and Doubts about Actions. The HMPS has three subscales: Self-oriented perfectionism; Socially-prescribed perfectionism; and Other-oriented perfectionism. When the scales are considered together a consistent two-factor solution has been found consisting of positive striving and maladaptive evaluative concerns (Bieling, Israeli and Antony, 2004), similar to the structure of perfectionism suggested by Hamachek (1978) who argued that there were two types of perfectionism, neurotic and normal. While there is substantial evidence linking the positive striving dimension of perfectionism with positive adjustment (Stoeber and Otto, 2006), the maladaptive evaluative concerns aspect of perfectionism is of interest to clinicians given its association with psychopathology. More recently Shafran, Cooper and Fairburn (2002) put forward a definition of a single construct of "clinical perfectionism (the over-dependence of self-evaluation on the determined pursuit of personally demanding, self-imposed standards in at least one highly salient domain, despite adverse consequences)" (p. 778), and Dunkley, Zuroff and Blankstein (2006) have suggested that self-criticism rather than perfectionism has an independent relationship with psychopathology.

Research evaluating cognitive behavioural therapy (CBT) for perfectionism with clinical samples has found significant reductions in perfectionism and negative affect. Glover, Brown, Fairburn and Shafran (2007) reported on a multiple baseline single case experimental design series with nine individuals with an anxiety disorder or depression. After receiving 10 sessions of CBT for clinical perfectionism, participants had clinically significant improvement on perfectionism as measured by the Clinical Perfectionism Questionnaire (Fairburn, Cooper and Shafran, unpublished, in Riley, Lee, Cooper, Fairburn and Shafran, 2007), FMPS and HMPS. Three participants also had clinically significant reductions in depression. Egan and Hine (2008) also found significant reductions in perfectionism on the FMPS in a single case experimental design series following an 8-session treatment for clinical perfectionism in individuals with anxiety and depression. A randomized-controlled trial investigating the treatment of clinical perfectionism versus a waitlist control found that the treatment group, who received 10 sessions of CBT for clinical perfectionism over 8 weeks, demonstrated clinically significant reductions in perfectionism across a number of measures in comparison 
to the control group (Riley et al., 2007). There were also significant reductions in anxiety and depression that were maintained at 16 week follow-up. Steele and Wade (2008) compared CBT for perfectionism with CBT for bulimia nervosa with a placebo condition in a sample of 48 patients. There were no significant differences between groups at post-treatment or followup on either eating- or psychopathology-related measures; however CBT for perfectionism produced larger effects for decreases in anxiety and depression than the other treatments, while still resulting in significant reductions in bulimic symptoms.

Egan et al. (2011) have argued these data are supportive of the notion of perfectionism being a transdiagnostic process, and that by targeting it in treatment a wide range of symptoms associated with Axis 1 disorders may be reduced. The mechanism of this action is unclear. The model of clinical perfectionism suggests that moderation occurs, whereby cognitive biases interact with perfectionism to increase psychopathology (Shafran et al., 2002), where different cognitive biases influence who develops different types of psychopathologies in response to perfectionism. For example, an all or nothing style of thinking may be more likely to lead to depressive disorders, whereas internalization of the thin ideal may result in disordered eating.

While the data suggest that individual treatment for perfectionism is effective in reducing perfectionism and negative affect (Egan and Hine, 2008; Glover et al., 2007; Riley et al., 2007; Steele and Wade, 2008), producing predominately large effects (Egan et al., 2011), less is known about the effective forms in which to deliver this treatment. While minimalist interventions such as guided self-help to target perfectionism have been shown to be effective (e.g. Pleva and Wade, 2006; Steele and Wade, 2008), less is known about the efficacy of brief psycho-education interventions about clinical perfectionism. This has been assessed by only one study (Aldea, Rice, Gormley and Rojas, 2010), which examined the efficacy of a feedback intervention on levels of emotional reactivity, symptomatic distress and self-esteem in adults with clinical perfectionism. Participants were randomized to either receive feedback on their perfectionism, using a technique adapted from Finn and Tonsager (1992) and Newman and Greenway (1997), or to be in a control condition that did not include this feedback. The feedback intervention produced significant reductions in symptomatic distress and emotional reactivity.

Additionally, to date as far as the authors are aware, there are no published data reporting on CBT for perfectionism delivered in a group therapy format. Research is therefore required to investigate the efficacy of targeting clinical perfectionism within a group format involving a larger sample with mixed diagnostic status. Group CBT has been demonstrated to be more efficient than individual CBT for patients presenting with obsessive compulsive disorder and coexisting psychopathology, with results finding equivalent change in symptom improvement, and group CBT showing lower attrition compared to individual therapy (Anderson and Rees, 2007). If group therapy targeting perfectionism is found to be of positive benefit, therapeutic delivery within a group setting will assist time constraints of clinicians and lead to more financially viable treatment access (Himle, Van Etten and Fischer, 2003). Additionally, given the hypothesized transdiagnostic function of perfectionism, it is important to examine the treatment with a mixed diagnostic group, to ensure its relevance for different clinical groups, which would support the parsimony of targeting perfectionism as opposed to a range of symptoms.

Therefore the aims of the current study were twofold. The first was to investigate the efficacy of psycho-education (information-alone) about clinical perfectionism in an outpatient psychiatric population with clinical levels of perfectionism and a variety of Axis 1 diagnoses. 
Based on previous research (Aldea et al., 2010) we hypothesized that an informationalone (psycho-education) condition would be superior to a waiting-list condition at both post-treatment and follow-up with respect to decreases in our primary outcome variable, perfectionism, and our secondary outcome variable, negative affect. The second aim was to investigate the efficacy of a group CBT for perfectionism. We also hypothesized that the treatment condition would be superior to both a waiting-list and an information-alone (psycho-education) condition at both post-treatment and follow-up in terms of reducing perfectionism and negative affect. Further, given the transdiagnostic nature of perfectionism, we hypothesized that group CBT for perfectionism would result in a reduction in Axis 1 symptomatology.

\section{Method}

\section{Participants}

The study was multi-site and was conducted at the Centre for Treatment of Anxiety and Depression in Adelaide, South Australia, and at the Curtin University Psychology Clinic in Perth, Western Australia, where the treatment programs were advertised. Inclusion criteria for the study required that participants were between 18 and 67 years old, had access to a General Practitioner or health professional capable of monitoring mental status, and reported high levels of perfectionism on the Concern over Mistakes subscale of the FMPS (Frost et al., 1990), using cut-offs suggested by previous research by Egan and Hine (2008) i.e. total score $\geqslant 22$ or mean item score $\geqslant 2.44$. Participants prescribed antidepressant medication were required to have been stable on medication for a minimum of at least 3 months. Exclusion criteria included current specialist treatment for perfectionism and severe psychiatric conditions that would interfere with treatment (e.g. active suicidality, major substance abuse or dependence, or psychosis). These conditions were assessed over the telephone and via completion of a screening questionnaire.

Initial phone screening was carried out with 47 people (21 at the Adelaide site, 26 at the Perth site), 12 (26\%) of whom were considered ineligible for the study for various reasons such as unable to be contacted $(n=4)$, made contact after the study cut-off date $(n=$ 2), a psychiatric condition that would interfere with treatment, e.g. psychosis, substance abuse, suicidality $(n=5)$, too distressed to participate $(n=1)$. A screening questionnaire package was then sent to 35 individuals, of whom $2(6 \%)$ were excluded from the study due to reporting a score below the cut-off for the Concern over Mistakes perfectionism questionnaire $(n=2)$, and a further 5 people $(14 \%)$ withdrew from the study with no reason given. Baseline appointments were then made for 28 individuals. A total of 21 participants (11 from Adelaide and 10 from Perth) were therefore entered into the study, with 19 participants (90.5\%) completing the 8-week CBT program (defined as attending at least 6 sessions). One participant from each site withdrew from the program. Treatment completers attended a mean of 7.01 sessions $(S D=1.64)$. Figure 1 presents a summary of the flow of participants through each stage of the study. The research was approved by the Central Northern Adelaide Health Service Ethics of Human Research Ethics Committee and the Curtin University Human Research Ethics Committee.

Using a series of independent samples $t$-tests, no significant differences were found among the two treatment sites on any of the baseline outcome measures. Therefore data from both 


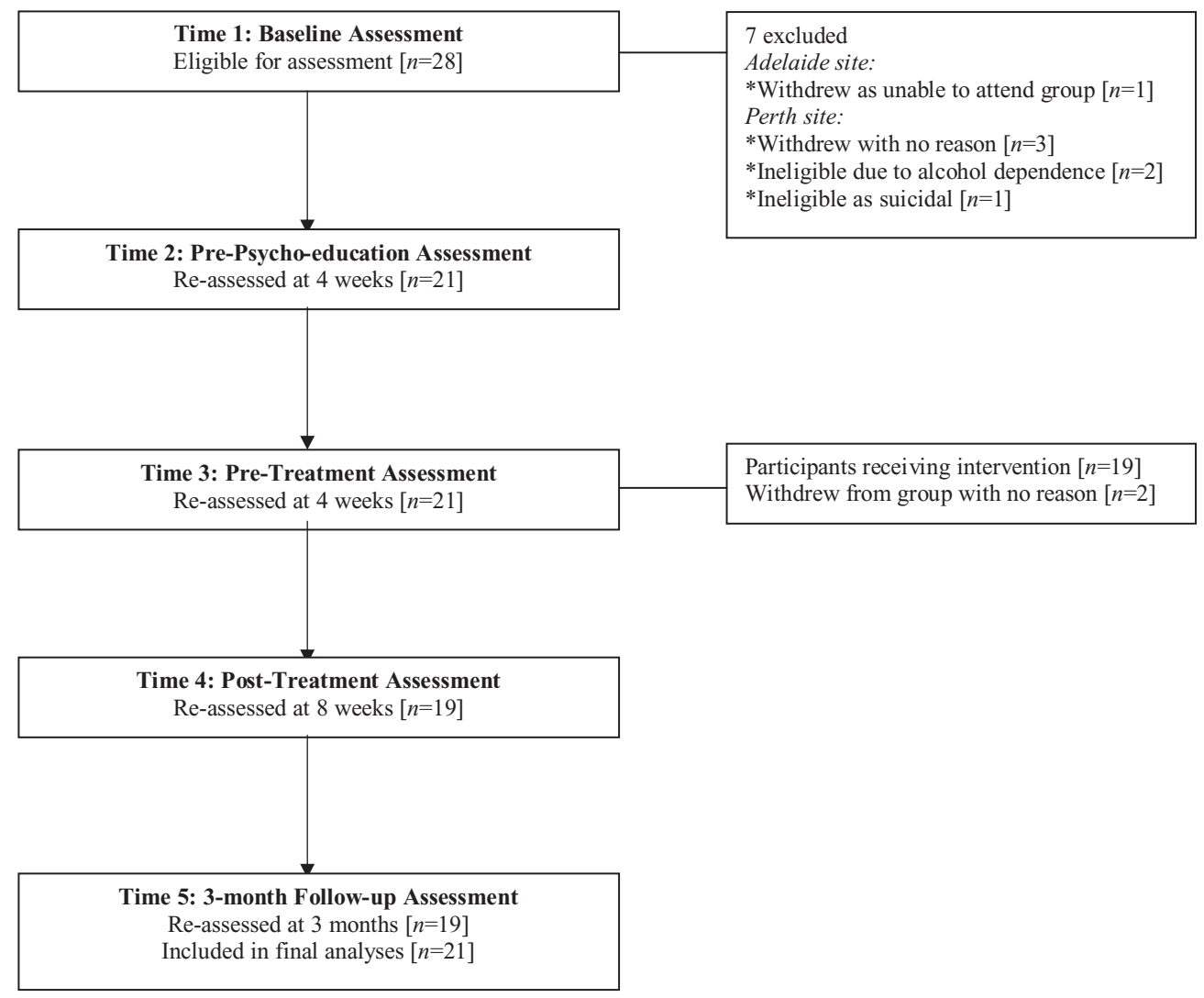

Figure 1. Flow of participants through each stage of the study

sites were pooled for the main analyses, and demographic and clinical characteristics are shown in Table 1. Of the 7 participants who did not meet criteria for a current Axis I disorder, 5 met criteria for depression in remission. The other 14 participants had current primary diagnoses of Major Depressive Episode $(n=4)$, Social Phobia $(n=3)$, Panic Disorder $(n=$ $2)$, Dysthymia $(n=2)$, Generalized Anxiety Disorder $(n=2)$, and Obsessive-Compulsive Disorder $(n=1)$ and 3 of the 14 met criteria for depression in remission. In addition, at baseline assessment 13 of the 21 participants in the study (62\%) were taking psychotropic medication for a mental health problem other than clinical perfectionism, 8 of whom were also receiving some form of psychotherapy, and 1 of whom was receiving psychotherapy and electroconvulsive therapy over the waiting-list period. Another 5 participants (24\%) were receiving psychotherapy alone for a mental health problem other than clinical perfectionism.

\section{Measures}

Screening questionnaires. While the intervention targeted clinical perfectionism, this measure has not been reported on much in the literature. Therefore entry criteria into the 
Table 1. Baseline demographic variables, clinical characteristics and outcome measures for the Adelaide group, Perth group and the combined sample $(N=21)$

\begin{tabular}{lc}
\hline & Combined sample $(n=21)$ \\
\hline Demographic variables: & 15 females, 6 males \\
Gender & $35.77(14.40)$, range $=18-67$ years \\
Age & $9(43 \%)$ single \\
Marital status & $19(91 \%)$ \\
English as first language & $11(52 \%)$ completed Bachelor degree or higher \\
Education status & $8(38 \%)$ full-time employed \\
Occupation & \\
Clinical characteristics: & $7(33 \%)$ without Axis I diagnosis (5 were in \\
Current Axis I disorder & remission from depression) \\
& $5(24 \%)$ with 1 Axis I diagnosis \\
& $9(43 \%)$ with 2+ Axis I diagnosis \\
& \\
Baseline outcome measures: & $2.40(0.41)$ \\
Clinical perfectionism & $3.60(0.75)$ \\
CM perfectionism & $4.04(0.56)$ \\
PS perfectionism & $4.40(1.30)$ \\
Self criticism & $1.04(0.81)$ \\
Depression & $0.67(0.61)$ \\
Anxiety & $1.44(0.66)$ \\
Stress &
\end{tabular}

Notes: $\mathrm{CM}=$ Concern over Mistakes, $\mathrm{PS}=$ Personal Standards. Mean item scores were used where higher scores are indicative of increased symptoms

study was measured using the 9-item Concern over Mistakes (CM) subscale of the FMPS (Frost et al., 1990) given its widespread use and more accessible comparability to other studies of perfectionism. The CM subscale has good reliability and internal consistency (Cronbach's $a=.88$; Frost et al., 1990). The Psychiatric Diagnostic Screening Questionnaire (PDSQ; Zimmerman and Mattia, 2001) was used to screen for psychiatric symptomatology. The PDSQ is a brief self-report scale that screens for 13 of the most common Axis I disorders encountered in outpatient mental health settings. A large-scale validation study within psychiatric outpatient settings has shown the PDSQ to have good sensitivity for detecting clinical cases (87\%), and high negative predictive value (97\%) in identifying healthy participants as non-cases (Zimmerman and Chalminski, 2006).

\section{Outcome measures}

Perfectionism. The 12-item Clinical Perfectionism Questionnaire (CPQ; unpublished in Riley et al., 2007) was used to assess clinical perfectionism over the past month using a 4-point Likert scale ranging from 1 (not at all) to 4 (all of the time). Participants were asked about report on the items (e.g. have you judged yourself on the basis of your ability to achieve high standards?) over the past week for the purpose of the current study. Steele, O'Shea, Murdock and Wade (2011) found evidence of convergent validity of the CPQ as it was significantly correlated with the FMPS subscales of Personal Standards $(r=.73)$ and CM 
( $r=.76$ ). Steele et al. found good internal consistency of the CPQ with $\alpha=.83$. In their recent validation study, Chang and Sanna (2012) found that scores on the CPQ were significantly correlated with symptoms of depression, anxiety, stress, negative affect, and multidimensional perfectionism; and that it contributed significant additional variance on each of their measures of depression, anxiety, and stress when controlling for negative affect and multidimensional perfectionism. The CPQ yielded high internal reliability $(\alpha=.83)$. Dickie and colleagues (Dickie, Surgenor, Wilson and McDowall, 2012) conducted a recent factor analytic study of the CPQ in a sample of 491 undergraduate students, and found a two-factor structure broadly reflecting positive striving and maladaptive evaluative concerns where both factors had modest internal consistency $(\alpha=.71)$. All of the items of the CPQ are listed in this latter study. In the current study the internal consistency was .70 .

In order to permit comparison with previous treatment studies, other measures of perfectionism were used, including the 9-item CM subscale and 7-item Personal Standards (PS) subscale from the FMPS (Frost et al., 1990), where participants were asked to indicate their agreement with the items using a 5-point Likert scale ranging from 1 (strongly disagree) to 5 (strongly agree). In the current study, good internal consistency was demonstrated: .91 (CM) and .79 (PS). The 15-item self-criticism subscale of the Dysfunctional Attitude Scale (DAS; Weissman and Beck, 1978) was also used to assess individuals' perceptions of themselves and others when their standards are not reached. Earlier analyses (Dunkley, Sanislow, Grilo and McGlashan, 2006) showed that self-criticism accounted fully for the relationship between perfectionism measures and depressive, anxious and eating disorder symptoms. Participants rated their agreement with items on a 7-point scale. It has previously been shown to have good reliability and validity (Oliver and Baumgart, 1985). For the current study, a Cronbach's alpha of .94 was obtained.

Axis 1 diagnoses. The Mini-International Neuropsychiatric Interview (MINI version 5.0.0; Sheehan et al., 1998) is a structured diagnostic interview used to assess for Axis I psychiatric disorders as defined by the Diagnostic and Statistical Manual of Mental Disorders ( $4^{\text {th }}$ ed. Text revision; DSM-IV-TR; American Psychiatric Association [APA], 2000). The MINI has good inter-rater and test-retest reliability (Lecrubier et al., 1997).

Negative affect. Symptoms of depression, anxiety and stress were measured using the 21item Depression Anxiety Stress Scale (DASS-21; Lovibond and Lovibond, 1995). For each item, participants were asked to rate how much a particular statement applied to them over the past week on a 4-point Likert scale ranging from 0 (did not apply to me at all) to 3 (applied to me very much, or most of the time). It has been shown to have excellent internal consistency and validity in both clinical and non-clinical samples (Antony, Bieling, Cox, Enns and Swinson, 1998). The present study found good internal consistency for the total score with a Cronbach's alpha of .88.

\section{Design and procedure}

Eligible participants were assessed at five time points using a structured diagnostic interview and self-report questionnaire. Following the baseline assessment (Time 1) participants were entered into a 4-week waiting-list period in order to provide their own no-treatment control condition. Participants were re-assessed pre-psycho-education (Time 2) and were then provided with chapters 1 to 4 from the self-help book Overcoming Perfectionism (Shafran, 
Egan and Wade, 2010). The reading material covered information on defining clinical perfectionism, understanding how it relates to different disorders, causes of perfectionism, and how perfectionism may be maintained. Participants were asked to read the material over the coming -4week period to determine the impact of psycho-education (information-alone condition). Participants were then re-assessed at the pre-treatment (Time 3) and commenced the 8-week group cognitive behaviour therapy (CBT) program. Within 2 weeks of completing the group program participants were re-assessed (Time 4) and then again 3 months later (Time 5).

\section{Group CBT for clinical perfectionism}

The group CBT program consisted of eight 2-hour sessions conducted over an 8-week period. The intervention was derived from the manual Overcoming Perfectionism (Shafran et al., 2010). The group sessions followed chapters 5 to 10 of the book. Participants were provided with readings and worksheets to complete each week for homework. A copy of the book was given to participants at the completion of the group program. Detailed session plans were developed and delivered across both sites to ensure adherence to the material. Session content included analysing costs and benefits of change, psycho-education, using surveys and behavioural experiments to challenge perfectionistic beliefs, introducing and identifying cognitive distortions and implementing cognitive restructuring techniques, targeting procrastination, problem solving, time-management, developing compassion for self and relapse prevention. This book was chosen for the intervention as it has not previously been evaluated whereas other similar books have been (e.g. Pleva and Wade, 2006).

Homework was assigned at the end of each session and reviewed as a group at the following session. Therapists collected homework to review for adherence. At the beginning of each group session (with the exception of session 1) homework compliance was evaluated using a measure adapted from previous trials (Thiels, Schmidt, Troop, Treasure and Garthe, 2001), assessing the amount of reading done, the proportion of exercises completed and the time spent on homework.

\section{Group therapists}

Two therapists delivered the group program at each site. At the Adelaide site, the treatment program was delivered by a psychologist (AS) and a psychiatrist (SW), whilst in Perth the treatment was delivered by two final-year postgraduate clinical psychology trainees (JE and $\mathrm{AH})$ under the supervision of a psychologist (SE).

\section{Statistical analyses}

All analyses were conducted using version 17.0 of the Statistical Package for the Social Sciences (SPSS). Multi-level mixed effects linear regression analyses were used to determine whether scores on each outcome measure significantly changed over time, as well as the specific time points at which statistically significant change occurred (Holden, Kelley and Agarwal, 2008). These analyses retain all participants in analyses regardless of missing data and provide more accurate estimates of group means for smaller samples (Rashbash, Steele, Browne and Prosser, 2004). Given that CBT for clinical perfectionism has previously 
produced large effects on clinical perfectionism ( $d=1.31$; Riley et al., 2007), it was predicted that CBT would produce results with a large effect size in the current study (Cohen, 1988). Clinically significant change for the measure of clinical perfectionism (which the intervention was designed to reduce) was calculated using a method outlined in a previous trial (Riley et al., 2007) indicating if the participants' post-treatment or 3-month follow-up score was at least two standard deviations lower than the sample's mean pre-treatment score.

\section{Results}

\section{Homework compliance}

Following the 4-week information-alone period, participants reported that, on average, they had read $75-100 \%$ of the reading material provided. On average, over the course of the 8week group program participants reported reading $75-100 \%$ of the readings assigned for homework, 75-100\% of the exercises that were assigned for homework, and spending a total of approximately 50 minutes each week on homework $(M=51.82$ minutes, $S D=46.42$ minutes).

\section{Comparison of the waitlist, psycho-education, and group CBT}

From baseline to post-treatment, main effects for time were observed for all measures of perfectionism, self-criticism, depression, anxiety and stress (Table 2), associated with large effect sizes $(d \geqslant 1.46)$. The effect size changes from pre- to post-treatment were all large as well $(\geqslant .92)$. Mixed models analyses indicated that significant change occurred only between Times 1, 2 and 3 compared to Time 4. Nevertheless, according to the criteria for clinically significant improvement, only 4 of the 19 treatment completers $(21 \%)$ were clinically significantly improved at post-group assessment, compared to 15 out of 20 (75\%) reported in a previous perfectionism treatment study (Riley et al., 2007), where the mean scores of on the CPQ decreased from 2.89 to 2.31 from pre- to post-test.

\section{Effects of group CBT for perfectionism at 3-month follow-up}

From baseline to 3-month follow-up, main effects for time were observed for all measures of perfectionism, self-criticism, depression, anxiety and stress (Table 2), associated with large effect sizes $(d s \geqslant 1.50)$. There was no significant change for any of the outcome measures between Time 4 and Time 5 (post-group to 3-month follow-up), indicating that the significant changes observed in the measures over the 8-week group CBT intervention were maintained at the 3-month follow-up. Six of the 19 treatment completers (32\%) were "clinically significantly improved" at the 3-month follow-up assessment: 2 of the 4 who had been improved at posttreatment and an additional 4 people.

\section{Axis I diagnoses}

At baseline 7 participants (33\%) did not meet criteria for an Axis I mental disorder, whilst at the 3-month follow-up assessment 11 participants (52\%) did not meet criteria for a current Axis I diagnosis. Three of the four individuals meeting criteria for major depressive 
Table 2. Multi-level mixed effects linear regression analyses reporting estimated marginal means (standard error) of outcome variables over time $(N=21)$

\begin{tabular}{|c|c|c|c|c|c|c|c|}
\hline Variable & $\begin{array}{l}\text { Time 1: } \\
\text { Baseline } \\
\text { Mean (SE) }\end{array}$ & $\begin{array}{l}\text { Time 2: } \\
\text { Pre-psycho- } \\
\text { education } \\
\text { Mean (SE) }\end{array}$ & $\begin{array}{l}\text { Time 3: } \\
\text { Pre-treatment } \\
\text { Mean (SE) }\end{array}$ & $\begin{array}{l}\text { Time 4: } \\
\text { Post-treatment } \\
\text { Mean (SE) }\end{array}$ & $\begin{array}{l}\text { Time 5: } \\
\text { 3-month } \\
\text { follow-up } \\
\text { Mean (SE) }\end{array}$ & $\begin{array}{l}\text { Baseline to } \\
\text { post-treatment } \\
F(p) \text { ES }\end{array}$ & $\begin{array}{l}\text { Baseline to } \\
\text { follow-up } \\
F(p) \mathrm{ES}\end{array}$ \\
\hline \multicolumn{8}{|c|}{ Constructs related to perfectionism } \\
\hline $\begin{array}{l}\text { Clinical } \\
\text { perfectionism }\end{array}$ & $2.38(0.09)^{\mathrm{a}}$ & $2.23(0.10)^{\mathrm{a}}$ & $2.28(0.10)^{\mathrm{a}}$ & $1.85(0.06)^{\mathrm{b}}$ & $1.76(0.06)^{\mathrm{b}}$ & $10.74(<.001) 1.55$ & $16.06(<.001) 1.90$ \\
\hline CM perfectionism & $3.56(0.24)^{\mathrm{a}}$ & $3.49(0.24)^{\mathrm{a}}$ & $3.51(0.25)^{\mathrm{a}}$ & $2.36(0.22)^{b}$ & $2.28(0.24)^{\mathrm{b}}$ & $14.41(<.001) 1.72$ & $12.80(<.001) 1.64$ \\
\hline PS perfectionism & $4.01(0.12)^{\mathrm{a}}$ & $3.87(0.14)^{\mathrm{a}}$ & $3.95(0.12)^{\mathrm{a}}$ & $3.30(0.09)^{\mathrm{b}}$ & $3.22(0.11)^{\mathrm{b}}$ & $17.72(<.001) 1.91$ & $17.88(<.001) 1.93$ \\
\hline Self criticism & $4.40(0.34)^{\mathrm{a}}$ & $4.30(0.34)^{\mathrm{a}}$ & $4.43(0.33)^{\mathrm{a}}$ & $2.87(0.29)^{\mathrm{b}}$ & $2.66(0.30)^{\mathrm{b}}$ & $10.47(<.001) 1.46$ & $10.76(<.001) 1.50$ \\
\hline \multicolumn{8}{|c|}{ Negative affect } \\
\hline $\begin{array}{l}\text { Depression, } \\
\text { anxiety stress }\end{array}$ & $1.06(0.11)^{\mathrm{a}}$ & $0.95(0.13)^{\mathrm{a}}$ & $0.94(0.13)^{\mathrm{a}}$ & $0.53(0.08)^{b}$ & $0.50(0.08)^{b}$ & $12.51(<.001) 1.59$ & $11.83(<.001) 1.55$ \\
\hline
\end{tabular}

Notes: $\mathrm{CM}=$ Concern over Mistakes. PS $=$ Personal Standards. ES $=$ effect size, Cohen's $d$. Different superscripts indicate significant differences across time periods. Mean item scores were used where higher scores are indicative of increased symptoms. The effect size change between Times 3 and 4 ([Mean Time 4-MeanTime 3]/SD Time 3) was 0.92 (clinical perfectionism), 1.42 (CM), 1.16 (PS), 1.25 (self-criticism), .98 (negative affect) 
episode at the pre-group assessment were no longer meeting criteria at the 3-month follow-up assessment, and seven participants meeting criteria for social phobia at baseline (as primary or secondary diagnosis) were all no longer meeting criteria for this disorder at the follow-up assessment.

\section{Discussion}

The current study investigated the impact of psycho-education and group CBT for clinical perfectionism in a community sample. With respect to our first aim, and contrary to predictions, the information-alone intervention did not have a significant impact on any outcome measure. In a previous trial of self-help CBT for perfectionism in a non-clinical sample, a pure self-help condition (where individuals are provided with a self-help manual to read without therapist intervention) was shown to be effective in reducing symptoms of perfectionism and associated symptoms (Pleva and Wade, 2006). In another nonclinical sample, providing individual feedback to non-clinical participants on their level of maladaptive perfectionism led to reductions in self-reported psychological distress and emotional reactivity (Aldea et al., 2010). However, psycho-education alone (in the absence of providing any CBT strategies or personalized feedback) may not be a sufficient intervention in a clinical sample.

With respect to our second aim, the 8-week CBT group intervention was associated with substantial reductions on all measures and these changes were maintained at the 3-month follow-up assessment and associated with large effect sizes. These findings are consistent with hypotheses and previous literature supporting the efficacy of CBT for clinical perfectionism (Egan and Hine, 2008; Glover et al., 2007; Riley et al., 2007; Pleva and Wade, 2006; Steele and Wade, 2008). It must also be noted that these findings may have been produced as a result of participants being primed by psycho-education. That is, the information-alone condition may have increased participants' readiness to partake in treatment and may have been partially responsible for producing effective treatment results from group CBT.

Associated with these findings, approximately $40 \%$ of the sample met diagnostic criteria for at least two current Axis I diagnoses at baseline, consistent with research that perfectionism is significantly related to co-morbidity (Bieling et al., 2004). At follow-up, three of the four individuals diagnosed with a Major Depressive Episode no longer met criteria and of the seven participants meeting criteria for social phobia at baseline (as a primary or secondary diagnosis), none met criteria.

Surprisingly, at the post-group assessment only $21 \%$ of participants met criteria for "clinically significant improvement" according to their change in scores on the clinical perfectionism measure, and $32 \%$ at the 3-month follow-up assessment, in comparison to $75 \%$ in a previous trial of individual CBT for clinical perfectionism (Riley et al., 2007). This inconsistency may be partly explained by the difference in baseline scores of clinical perfectionism in the two samples - participants in the Riley et al. study reported higher baseline scores on the CPQ measure in comparison to this study. It may also represent the need for more sessions as participants in the aforementioned study received 10 sessions over 8 weeks versus 8 sessions over 8 weeks in the present study.

Overall, results indicate that group therapy for perfectionism is feasible and effective in reducing both perfectionism and negative affect. The advantages of group therapy relative to individual therapy include greater time efficiency and lower costs (Egan and Stout, 
2007; Himle et al., 2003). Group treatment also provides an opportunity for both peer and professional feedback and support. The attrition rate in this trial $(9.5 \%)$ is commensurate with previous trials of individual treatment for clinical perfectionism (e.g. Riley et al., 2007). Previous studies have shown that receiving treatment within a group reduces attrition in comparison to individual therapy in an OCD population (Anderson and Rees, 2007), and it would be of interest to investigate whether group therapy for perfectionism as an initial transdiagnostic treatment increases retention in subsequent individual therapies that might be more specifically tailored for a certain psychopathology.

However, these conclusions should be tempered by the limitations of the present study. First, the design of the study did not allow for a comparison group to control for nonspecific effects. Given that all participants received the information-alone condition prior to the group CBT for perfectionism intervention, one cannot disentangle whether the results were due solely to the group program or to an accumulative impact of the two interventions, or some other confounding variable. Second, 33\% of the sample did not meet criteria for a current Axis I diagnosis, which limits the generalizability of these findings to "pure" clinical samples. Third, while participants were excluded if receiving concurrent specialist treatment for perfectionism, it is not possible to know if (a) the individual was receiving input on their perfectionism as a part of any concurrent treatment for other issues, or (b) concurrent psychotherapy or treatments outside of the group had a beneficial impact on perfectionism, creating a possible confound in the results. Finally, it must be kept in mind that this intervention was only given to people with elevated levels of perfectionism and will not be helpful or relevant to all people experiencing difficulty with psychopathology. Previous results suggest that people experiencing elevated levels of perfectionism will receive significantly more benefit for their eating disorder if perfectionism is targeted than when it is ignored (Fairburn et al., 2009). It would be of interest to investigate whether this same finding exists for other types of psychopathology.

In summary, this represents the first study to demonstrate that a group format of treatment for clinical perfectionism was associated with decreases of large effect size in perfectionism and negative affect in a community sample with a range of illness severity. The findings also support the use of CBT strategies to target perfectionism, but not the use of psycho-education alone. Future research should examine the interplay between group and individual therapy in terms of producing an optimal outcome with respect to clinical significance for clients, and also how treatment of perfectionism performs against currently established and efficacious therapies for Axis 1 disorders, and also whether treating perfectionism in addition to targeted treatments for these disorders will substantially improve outcome.

\section{References}

Aldea, M., Rice, K., Gormley, B. and Rojas, A. (2010). Telling perfectionists about their perfectionism: effects of providing feedback on emotional reactivity and psychological symptoms. Behaviour Research and Therapy, 48, 1194-1203.

American Psychiatric Association (2000). Diagnostic and Statistical Manual of Mental Disorders, Text Revision, 4th ed. Washington, DC: American Psychiatric Association.

Anderson, R. A. and Rees, C. S. (2007). Group versus individual cognitive-behavioural treatment for obsessive-compulsive disorder: a controlled trial. Behaviour Research and Therapy, 45, 123-137. 
Antony, M., Bieling, P., Cox, B., Enns, M. and Swinson, R. (1998). Psychometric properties of the 42-item and 21-item versions of the Depression, Anxiety Stress Scales in clinical groups and a community sample. Psychological Assessment, 10, 176-181.

Antony, M. M., Purdon, C. L., Huta, V. and Swinson, R. P. (1998). Dimensions of perfectionism across the anxiety disorders. Behaviour Research and Therapy, 36, 1143-1154.

Beevers, C. G. and Miller, I. W. (2004). Perfectionism, cognitive bias and hopelessness as prospective predictors of suicidal ideation. Suicide and Life-Threatening Behavior, 34, 126-137.

Bieling, P. J., Israeli, A. L. and Antony, M. M. (2004). Is perfectionism good, bad, or both? Examining models of the perfectionism construct. Personality and Individual Differences, 36, 1373-1385.

Blatt, S. J. and Zuroff, D. (2005). Empirical evaluation of the assumptions in identifying evidence based treatments in mental health. Clinical Psychology Review, 25, 459-486.

Chang, E. C. and Sanna, L. J. (2012). Evidence for the validity of the Clinical Perfectionism Questionnaire in a nonclinical population: more than just negative affectivity. Journal of Personality Assessment, 94, 102-108.

Cohen, J. (1988). Statistical Power for the Behavioural Sciences, 2nd ed. Hillsdale, NJ: Erlbaum.

Dickie, L., Surgenor, L. J., Wilson, M. and McDowall, J. (2012). The structure and reliability of the Clinical Perfectionism Questionnaire. Personality and Individual Differences, doi:10.1016/j.paid.2012.02.003.

Dunkley, D., Sanislow, C., Grilo, C. and McGlashan, T. (2006). Perfectionism and depressive symptoms three years later: negative social interactions, avoidant coping and perceived social support as mediators. Comprehensive Psychiatry, 47, 106-115.

Dunkley, D., Zuroff, D. C. and Blankstein, K. R. (2006). Specific perfectionism components versus self-criticism in predicting maladjustment. Personality and Individual Differences, 40, 665-676.

Egan, S. J. and Hine, P. (2008). Cognitive behavioural treatment of perfectionism: a single case experimental design series. Behaviour Change, 25, 245-258.

Egan, S. J. and Stout, S. (2007). Group Cognitive Behavioural Treatment of Perfectionism. Invited paper presented in symposium, OCD and related cognitive styles: outcomes and treatment (Chair: Mike Kyrios, Discussant: Paul Salkovskis). World Psychiatric Association International Congress, Melbourne, Australia, November.

Egan, S. J., Wade, T. D. and Shafran, R. (2011). Perfectionism as a transdiagnostic process: a clinical review. Clinical Psychology Review, 31, 203-212.

Fairburn, C. G., Cooper, Z., Doll, H. A., O'Connor, M. E., Bohn, K., Hawker, D. M., et al. (2009). Transdiagnostic cognitive-behavioral therapy for patients with eating disorders: a two-site trial with 60-week follow-up. American Journal of Psychiatry, 166, 311-319.

Finn, S. and Tonsager, E. (1992). Therapeutic effects of providing MMPI-2 test feedback to college students awaiting therapy. Psychological Assessment, 4, 278-287.

Frost, R., Marten, P., Lahart, C. and Rosenblate, R. (1990). The dimensions of perfectionism. Cognitive Therapy and Research, 14, 449-468.

Frost, R. O., Novara, C. and Rhéaume, J. (2002). Perfectionism in obsessive-compulsive disorder In R. O. Frost and G. Steketee (Eds.), Cognitive Approaches to Obsessions and Compulsions (pp. 91-105). New York: Pergamon.

Frost, R. O. and Steketee, G. (1997). Perfectionism in obsessive-compulsive disorder patients. Behaviour Research and Therapy, 35, 291-296.

Glover, D., Brown, G., Fairburn, C. and Shafran, R. (2007). A preliminary evaluation of cognitive behaviour therapy for perfectionism: a case series. Journal of British Clinical Psychology, 46, 85-94

Hamachek, D. E. (1978). Psychodynamics of normal and neurotic perfectionism Psychology: A Journal of Human Behaviour, 15, 27-33.

Hawley, L. L., Ho, M. R., Zuroff, D. C. and Blatt, S. J. (2006). The relationship of perfectionism, depression and therapeutic alliance during treatment for depression: latent difference score analysis. Journal of Consulting and Clinical Psychology, 74, 930-942. 
Hewitt, P. L. and Flett, G. L. (1991). Perfectionism in the self and social contexts: conceptualisation, assessment and association with psychopathology. Journal of Personality and Social Psychology, 60, 456-470.

Himle, J. A., Van Etten, M. and Fischer, D. J. (2003). Group cognitive behavioral therapy for obsessive-compulsive disorder (OCD): a controlled study. International Journal of Psychiatry in Clinical Practice, 9, 257-263.

Holden, J. E., Kelley, K. and Agarwal, R. (2008). Analysing change: a primer on multilevel models with applications to nephrology. American Journal of Nephrology, 28, 792-801.

Juster, H. R., Heimberg, R. G., Frost, R. O., Holt, C. S., Mattia, J. I. and Faccenda, K. (1996). Social phobia and perfectionism. Personality and Individual Differences, 21, 403-410.

Lecrubier, Y., Sheehan, D. V., Weiller, E., Amorim, P., Bonora, I., Sheehan, K., et al. (1997). The mini international neuropsychiatric interview (MINI). A short diagnostic structured interview: reliability and validity according to the CIDI. European Psychiatry, 12, 224231.

Lovibond, P. F. and Lovibond, S. H. (1995). The structure of negative emotional states: comparison of the depression and anxiety inventories. Behaviour Research and Therapy, 33, 335-343.

Lundh, L. G. and Ost, L. G. (2001). Attentional bias, self-consciousness and perfectionism in social phobia before and after cognitive-behaviour therapy. Scandinavian Journal of Behaviour Therapy, 30, 4-16.

Newman, M. and Greenway, P. (1997). Therapeutic effects of providing MMPI-2 test feedback to clients at a university counseling service: a collaborative approach. Psychological Assessment, 9, $122-131$.

Oliver, J. M. and Baumgart, E. P. (1985). The Dysfunctional Attitude Scale: psychometric properties and relation to depression in an unselected adult population. Cognitive Therapy and Research, 9, 161-167.

Pleva, J. and Wade, T. (2006). Guided self-help versus pure self-help for perfectionism: a randomised controlled trial. Behaviour Research and Therapy, 45, 849-861.

Rashbash, J., Steele, F., Browne, W. and Prosser, B. (2004). A User's Guide to MLwiN. University of Bristol: Centre for Multilevel Modelling.

Riley, C., Lee, M., Cooper, Z., Fairburn, C. and Shafran, R. (2007). A randomised controlled trial of cognitive-behaviour therapy for clinical perfectionism: a preliminary study. Behaviour Research and Therapy, 45, 2221-2231.

Rosenberg, M. (1965). Society and the Self-Image. Princeton, NJ: Princeton University Press.

Sassaroli, S., Lauro, L. J., Ruggiero, G. M., Mauri, M. C., Vinai, P. and Frost, R. (2008). Perfectionism in depression, obsessive compulsive disorder and eating disorders.Behaviour Research and Therapy, 46, 757-765.

Shafran, R., Cooper, Z. and Fairburn, C. (2002). Clinical perfectionism: a cognitive behavioural analysis. Behaviour Research and Therapy, 40, 773-791.

Shafran, R., Egan, S. and Wade, T. (2010). Overcoming Perfectionism: a self-help manual using cognitive-behavioural techniques. London: Robinson.

Sheehan, D. V., Janavs, J., Baker, R., Sheehan, K. H., Knapp, E. and Sheehan, M. (1998). Mini International Neuropsychiatric Interview. Tampa: University of South Florida.

Steele, A. L., O'Shea, A., Murdock, A. and Wade, T. D. (2011). Perfectionism and its relation to overevaluation of weight and shape and depression in an eating disorder sample. International Journal of Eating Disorders, 44, 459-464.

Steele, A. and Wade, T. (2008). A randomised trial investigating guided self-help to reduce perfectionism and its impact on bulimia nervosa: a pilot study. Behaviour Research and Therapy, 46, 1316-1323.

Stoeber, J. and Otto, K. (2006). Positive conceptions of perfectionism: approaches, evidence, challenges. Personality and Social Psychology Review, 10, 295-319. 
Thiels, C., Schmidt, U., Troop, N., Treasure, J. and Garthe, R. (2001). Compliance with a self-care manual in guided self-change for bulimia nervosa. European Eating Disorders Review, 9, 115-122.

Tykra, A. R., Waldron, I., Graber, J. A. and Brooks-Gunn, J. (2002). Prospective predictors of the onset of anorexic and bulimic syndromes. International Journal of Eating Disorders, 32, 282-290.

Vohs, K. D., Bardone, A. M., Joiner, T. E., Abramson, L. Y. and Heatherton, T. F. (1999). Perfectionism, perceived weight status, and self-esteem interact to predict bulimic symptoms: a model of bulimic symptom development. Journal of Abnormal Psychology, 108, 695-700.

Weissman, A. N. and Beck, A. T. (1978). Development and Validation of the Dysfunctional Attitudes Scale: a preliminary investigation. Paper presented at the Annual Meeting of the American Educational Research Association, Toronto, Canada.

Zimmerman, M. and Chelminski, I. (2006). A scale to screen for DSM-IV axis I disorders in psychiatric outpatients: performance of the Psychiatric Diagnostic Screening Questionnaire. Psychological Medicine, 36, 1601-1611.

Zimmerman, M. and Mattia, J. (2001) A self-report scale to help make psychiatric diagnoses. Archives of General Psychiatry, 58, 787-794. 